\title{
Politique de la FMH en matière de médicaments
}

Des représentants des différents groupes intéressés ainsi que des experts ont participé à un séminaire organisé par la FMH sur la politique des médicaments.

Les aspects aussi bien scientifiques que politiques des traitements médicamenteux et de leurs ventes ont été abordés.

Dans le contexte actuel des discussions sur le coût du système de la santé, il n'est guère étonnant que le coût des médicaments ait été au centre du débat. Différentes options permettant de nouvelles économies par l'introduction de mesures de concurrences adéquates dans le domaine de la distribution des médicaments ont été discutées. Des modèles tant suisses qu'étrangers montrent que la concurrence peut être stimulée par le biais de nouveaux leviers et aussi que la responsabilité personnelle des consommateurs peut être renforcée. Les avis sont partagés, respectivement il n'est pas clairement établi, de savoir si la dispensation directe des médicaments par les médecins entraîne une hausse ou une diminution du coût des médicaments. Bien que la dispensation directe des médicaments par les médecins soit en soi une incitation à prescrire davantage, il est probable que cet inconvénient est compensé par une meilleure observance du traitement et par la prescription de médicaments LS plus avantageux.

En même temps le retrait du médicament Vioxx a rappelé le problème de la sûreté des médicaments. Une étude effectuée à l'hôpital universitaire de Zürich et l'hôpital cantonal de St-Gall présentée par Mme PD Dr Karin Fattinger a mis en évidence des effets secondaires médicamenteux chez environ 11\% des patients: Environ $4 \%$ des hospitalisations sont dues a un tel effet secondaire et le taux d'effet secondaire développé durant l'hospitalisation est de 7,5\%. Cet effet secondaire indésirable s'est avéré fatal pour $0,21 \%$ des patients hospitalisés pour effet secondaire ou ayant développé un effet secondaire durant l'hospitalisation. L'incidence d'effets secondaires développés en milieu hospitalier était de $0,4 \%$ pour ceux dus à une faute médicale et de $7,1 \%$ pour ceux survenus de manière imprévisi- ble. Pour les hospitalisations en raison effets secondaires, elles étaient dues dans 1,1\% des cas à des effets secondaires par faute médicale et dans 2,9\% des cas à des effets secondaires imprévisibles.

Les étiologies les plus fréquentes d'erreur étaient les contre-indications et les interactions non remarquées ainsi que les posologies non adaptées à la situation individuelle. Ces éléments soulignent l'importance primordiale d'être informé en détail sur les co-médications, les contreindications et les maladies concomitantes pour adapter le traitement médicamenteux à la situation individuelle. Un dossier électronique avec enregistrement des co-morbidités et une prescription électronique pourraient former un filet de sécurité permettant de repérer à temps les fautes potentielles et d'améliorer ainsi la sécurité des patients.

Aucune des personnes présentes n'a pu démontrer comment conjuguer les exigences de diminution des coûts en accroissant simultanément la sécurité des patients. Un consensus semble tout de même se dessiner pour affirmer que les obstacles à la concurrence devraient être éliminés à tous le niveaux de le formation des prix des médicaments.

Du point de vue de la FMH, ont peut tirer les conclusions suivantes - et provisoires! - de ce séminaire:

- Le patient doit être informé par son médecin des effets souhaités et des effets secondaires indésirables du traitement proposé. Lors du choix d'un traitement, il faut également tenir compte du rapport qualité/prix.

- Le patient (= client) devrait pouvoir choisir entre différentes propositions. Il devrait pouvoir également décider lui-même s'il veut acheter le médicament chez le médecin ou à la pharmacie.

- Le patient devrait pouvoir juger par luimême les standards de qualité et de sécurité lors du choix et de la vente des médicaments.

Dr Markus Trutmann, rédacteur en chef du Bulletin des médecins suisses 